\section{An esophageal ulcer mimicking advanced esophageal cancer in a patient on alendronate sodium treatment for osteoporosis}

We report a case of an esophageal ulcer that mimicked advanced esophageal cancer in a patient who was on alendronate sodium treatment.

A 56-year-old woman presented with progressively worsening pain on swallowing. For the past 6 months she had been taking $5 \mathrm{mg} /$ day alendronate sodium (Bonalon) for the treatment of postmenopausal osteoporosis. Upper gastrointestinal endoscopic examination revealed an ulcerative lesion in the lower esophagus that resembled an advanced esophageal cancer (Figure 1). The ulcer's mucosa was normal, but the ulcer bed was uneven and would not stain with Lugol dye. Biopsy specimens of the ulcer bed showed a marked infiltration of neutrophils and eosinophils in the subepithelial space, but no signs of malignancy (Figure 2). We stopped the alendronate sodium and prescribed a proton-pump inhibitor. Endoscopic examination 2 months later showed marked improvement in the ulcer, with no esophageal stricture.

This is the first report, to the best of our knowledge, of an alendronate-induced esophageal ulcer mimicking advanced esophageal cancer. Alendronate sodium, a bisphosphonate that inhibits osteoclast activity, is principally used to prevent and treat osteoporosis and is generally considered to be well tolerated. One multinational, randomized, double-blind study, in which postmenopausal women with osteoporosis were treated with alendronate for up to 10 years, concluded that the drug's adverse effects on the upper gastrointestinal tract were similar to those of placebo [1]. However, several previously reported cases of severe esophagitis and perforation of the esophagus suggest that adverse effects of alendronate might be more common than is sometimes recognized [2].

We should be aware of the possibility of esophageal lesions resulting from the use of bisphosphonates in patients with os-

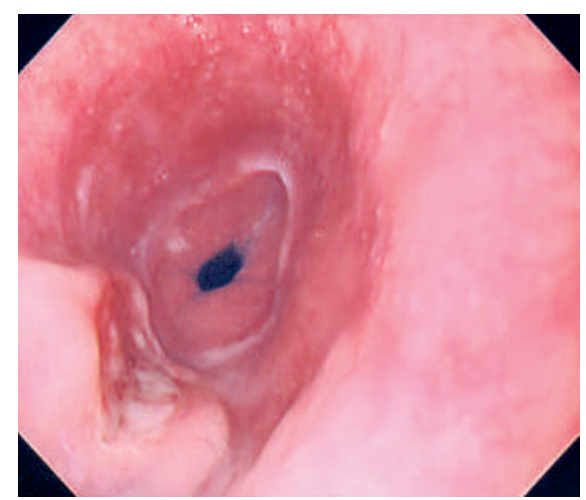

Figure 1 Endoscopic image of the lower esophagus showing an ulcerative lesion mimicking advanced esophageal cancer.

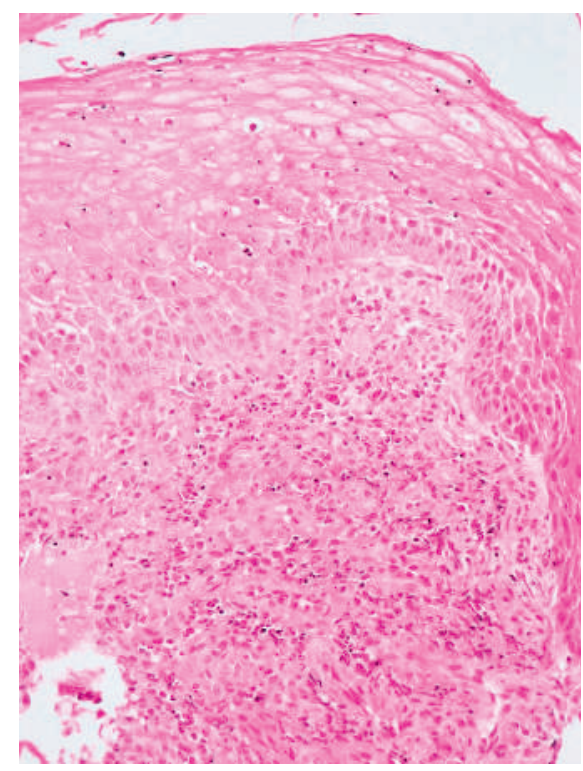

Figure 2 Histopathological examination of a biopsy specimen taken from the ulcer bed revealed a marked infiltration of neutrophils and eosinophils in the subepithelial space.

teoporosis. If a patient taking alendronate presents with acute-onset dysphagia or heartburn, an upper gastrointestinal endoscopic examination should be considered in order to exclude ulceration.

Endoscopy_UCTN_Code_CCL_1AB_2AC_3AZ
T. Terashima, K. Hiramatsu,

A. Shimatani, M. Matsuda, H. Ogino, Y. Satomura, Y. Noda

Department of Internal Medicine, Toyama Prefectural Central Hospital, Toyama, Japan.

\section{References}

${ }^{1}$ Bone HG, Hosking D, Devogelaer JP et al. Ten years' experience with alendronate for osteoporosis in postmenopausal women. Alendronate Phase III Osteoporosis Treatment Study Group. N Engl J Med 2004; 350: 1189-1199

2 Ryan JM, Kelsey P, Ryan BM, Mueller PR. Alendronate-induced esophagitis: case report of a recently recognized form of severe esophagitis with esophageal stricture: radiographic features. Radiology 1998; 206: $389-391$

\section{Corresponding author}

\section{Y. Noda, M.D.}

Department of Internal Medicine Toyama Prefectural Central Hospital 2-2-78 Nishinagae

Toyama

Toyama Prefecture 930-8550

Japan

Fax: $\quad$ +81-76-422-0667

E-mail: tera@medf.m.kanazawa-u.ac.jp 